\title{
Dapagliflozina mejoraría desenlaces clínicos en pacientes con insuficiencia cardiaca y fracción de eyección reducida
}

\author{
Dapaglifozin could improve clinical outcomes in patients with heart failure and reduced eyection fraction
}

\section{Comentado de:}

McMurray JJV et al. N Engl J Med 2019; 381:1995-2008. PMID: $31535829^{1}$

\section{Objetivos}

Evaluar la eficacia y seguridad de la dapagliflozina, un inhibidor del cotransportador de sodio-glucosa tipo 2 (SGLT-2) en pacientes con insuficiencia cardiaca (IC) y fracción de eyección (FE) reducida, independientemente de la presencia de diabetes.

\section{Diseño, lugar y participantes}

Ensayo clínico aleatorizado, multicéntrico en fase 3, controlado con placebo. El estudio incluyó 4.744 pacientes con IC en clase funcional II-IV de la NYHA y FE menor o igual a $40 \%$. Los pacientes además debían tener niveles elevados de NT-pro BNP (fragmento $\mathrm{N}$-terminal del péptido natriurético tipo $\mathrm{B}$ ).

Se excluyeron los pacientes con efectos adversos recientes ocasionados por algún SGLT-2, quienes padecieran diabetes mellitus tipo 1, tuvieran una presión arterial sistólica menor a 95 $\mathrm{mm} \mathrm{Hg}$ y una tasa de filtrado glomerular menor de $30 \mathrm{ml} / \mathrm{minu}$ to/1.73 $\mathrm{m}^{2}$

\section{Intervención}

Se aleatorizó a los participantes a recibir dapagliflozina en dosis de $10 \mathrm{mg} /$ día o placebo, además del tratamiento recomendado para la IC.

\section{Medición de resultados principales}

El punto final primario fue definido como el empeoramiento de la IC (internación o consulta que requiriese diuréticos endoveno- sos) o muerte cardiovascular.

\section{Resultados}

Después de una mediana de seguimiento de 18,2 meses, el evento primario ocurrió en $16,3 \%$ de los participantes en el grupo intervención y en $21,2 \%$ de los pacientes en el placebok, con un Hazard Ratio (HR) estimado de 0,74 (Intervalo de confianza [IC] del $95 \%, 0,65$ a 0,$85 ; p<0,001)$. El empeoramiento por IC ocurrió en $10 \%$ de los pacientes con dapagliflozina y en $13,7 \%$ de quienes recibieron placebo (HR 0,70; IC 95\%, 0,59 a 0,83) La muerte cardiovascular se presentó en $9,6 \%$ participantes del grupo dapagliflozina y $11,5 \%$ del placebo (HR 0,82; IC 95\%, 0,69 a 0,98$)$. No se observaron diferencias significativas en relación a la ocurrencia de episodios de hipovolemia, hipoglucemia severa ni de insuficiencia renal.

\section{Conclusión}

En los pacientes con IC y FE reducida, el riesgo de empeoramiento de la IC y la muerte cardiovascular fue más bajo en los pacientes tratados con dapagliflozina que en los tratados con placebo, independientemente de la presencia de diabetes.

Fuente de financiamiento/Conflicto de interés de los autores: Estudio financiado por AstraZeneca. Un resumen y los formularios completos de cada autor informando sus conflictos de interés se encuentran disponibles en la publicación original.

\section{Comentario}

Los hallazgos de este estudio, el Dapagliflozin and Prevention of Adverse Outcomes in Heart Failure (DAPA-HF, por sus iniciales en inglés), muestran que la dapagliflozina es una droga eficaz y segura en los pacientes con IC y FE reducida. En relación al beneficio clínico, sería necesario tratar a 21 pacientes para reducir un evento combinado sin exceso de eventos adversos peligrosos. El efecto favorable se observó en todos los subgrupos evaluados (etiología de la IC, fibrilación auricular y diabetes, entre los más importantes). Hay que destacar que los pacientes de este estudio fueron tratados de acuerdo a las recomendaciones de las guías internacionales, de modo que el agregado de dapagliflozina se asoció un beneficio adicional al del cuidado habitual.

Entre los mecanismos terapéuticos propuestos, se postulan: reducción de la precarga y de la postcarga, efectos metabólicos sobre el miocardio y protección renal. El beneficio precoz -antes de los 3 meses- hace pensar que el efecto diurético podría ser dominante. En el seguimiento los pacientes tratados con dapagliflozina se observaron valores de creatinina más bajos y hematocrito más elevados que en los tratados con placebo.

La dapagliflozina es un hipoglucemiante y junto a otras drogas de la familia de los SGLT-2 había demostrado en estudios previos reducir los eventos cardiovasculares (principalmente la IC), en pacientes con diabetes y $\sin$ IC previa $^{2-4}$.

\section{Conclusiones del comentador}

En función de los resultados descritos y la relación riesgo-beneficio, es probable que las guías de práctica clínica incorporen rápidamente a la dapagliflozina dentro del tratamiento estándar de la insuficiencia cardíaca.

Aníbal Arias [ Unidad Coronaria, Servicio de Cardiología, Hospital Italiano de Buenos Aires. anibal.arias@hospitalitaliano.org.ar ]

Arias A Dapagliflozina mejoraría desenlaces clínicos en pacientes con insuficiencia cardiaca y fracción de eyección reducida. Evid Actual Pract Ambul. 2020;23(1):e002044. Comentado de: McMurray JJV et al. Dapagliflozin in Patients with Heart Failure and Reduced Ejection Fraction. N Engl J Med 2019 381:1995-2008. PMID: 31535829 


\section{Referencias}

1. McMurray JJV, Solomon SD, Inzucchi SE, et al. Dapagliflozin in Patients with Heart Failure and Reduced Ejection Fraction. N Engl J Med. 2019;381(21):1995-2008. Available from: 10.1056/NEJMoa1911303.

2. Zinman B, Wanner C, Lachin JM, et al. Empagliflozin, Cardiovascular Outcomes, and Mortality in Type 2 Diabetes. N Engl J Med. 2015;373(22):21172128. Available from: $10.1056 /$ nejmoa1504720

3. Wiviott SD, Raz I, Bonaca MP, et al. Dapagliflozin and Cardiovascular Outcomes in Type 2 Diabetes. N Engl J Med. 2019;380(4):347-357. Available from: 10.1056/nejmoa1812389.

4. Neal B, Perkovic V, Mahaffey KW, et al. Canagliflozin and Cardiovascular and Renal Events in Type 2 Diabetes. N Engl J Med. $2017 ; 377(7): 644-657$. Available from: 10.1056/nejmoa1611925. 